\title{
Limited cross reactivity among arginine kinase allergens from mealworm and cricket edible insects
}

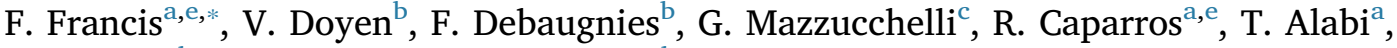 \\ C. Blecker ${ }^{\mathrm{d}, \mathrm{e}}$, E. Haubruge ${ }^{\mathrm{a}, \mathrm{e}}$, F. Corazza ${ }^{\mathrm{b}, *}$ \\ a Functional and Evolutionary Entomology, Gembloux Agro-Bio Tech, University of Liege, Passage des Deportes-2, B-5030 Gembloux, Belgium \\ ${ }^{\mathrm{b}}$ CHU Brugman, Immunology IRIS Laboratory, Belgium \\ ${ }^{\mathrm{c}}$ Mass Spectrometry Laboratory, University of Liege, Belgium \\ ${ }^{\mathrm{d}}$ Food Science and Formulation, Gembloux Agro-Bio Tech, University of Liege, Passage des Deportes-2, B-5030 Gembloux, Belgium \\ e TERRA Research and Teaching Center, Gembloux Agro-Bio Tech, University of Liege, Passage des Deportes-2, B-5030 Gembloux, Belgium
}

A R T I C L E I N F O

\section{Keywords:}

Insect allergen

Proteomics

Arginine kinase

Purification

Western blot

\begin{abstract}
A B S T R A C T
Insects are seen as a solution to the increasing demand for protein sources for food. However, entomophagy has unfortunately been linked to allergic reactions in Europe with people with professional contacts. As mealworms (Tenebrio molitor) and crickets (Acheta domesticus) have recently become commercially available (both whole or in food formulation) in several European countries, this research assessed the cross allergenicity of arginine kinase (AK). Based on the collection of sera from a entomology laboratory staff, oven cooked insects but also purified AK fractions were tested. Immunoblotting against the protein extracts revealed different Immunoglobulin E reactivity of sera according to the insect target species: two bands (40 and $14 \mathrm{kDa}$ ) for crickets and a pattern including light responses at 17, 25 and $37 \mathrm{kDa}$ for mealworms. Focusing on $\mathrm{AK}$, low specific allergenicity was here illustrated and discussed in relation to the development of a safe edible insect consumption by humans.
\end{abstract}

\section{Introduction}

Insects appear increasingly as a future solution as animal resource for human food (Belluco et al., 2013; FAO, 2009; Gahukar, 2011). Despite been considered delicacies in many tropical countries (van Huis, 2003), edible insects are seen by Western populations as an aberration, as consumption is less consistent with their eating habits or even as a threat to their country cultural and psychological identity (Caparros et al., 2013; Looy, Dunkel, \& Wood, 2013; Ramos-Elorduy, 2009; van Huis, 2003).

Currently, 2086 species of insects are consumed in 130 countries (Ramos-Elorduy, 2009; Rumpold \& Schlüter, 2013). In Belgium, the Federal Agency for the Safety of the Food Chain (FASFC) published a list of ten insect species allowed for consumption with conventional requirements for food safety (FASCS, 2013). Among these 10 species, crickets (Acheta domesticus (L. 1758); Orthoptera: Gryllidae) and mealworm larvae (Tenebrio molitor L. 1758; Coleoptera: Tenebrionidae) are the most promising and the most reared in Europe. Several small companies specializing in the breeding and integration of edible insects in food products have gradually emerged. If properly managed and consumed, edible insects are considered safe for human consumption and extremely beneficial when other classical food recommendation (e.g. food portion and balanced diet) are respected (Belluco et al., 2013; Sogari, Menozzi, \& Mora, 2017). Nevertheless, as insects are closely related to Crustaceans and Acari, the probability of an allergic reaction associated with entomophagy is strongly suggested and the main responsible allergens were recently identified (e.g. tropomyosine, $\alpha$ amylase or aginine kinase) (Barre et al., 2014, 2016; Verhoeckx et al., 2013). A preliminary belgian study showed that several exposed subjects $(19 \%)$ were found to be sensitized by skin prick tests made with delipidated and grilled insect samples from T. molitor and A. domesticus (Mairesse et al., 2014).

According to the significant sensitization observed toward cricket and mealworm samples during this first investigation, further works

\footnotetext{
* Corresponding authors at: Functional and Evolutionary Entomology, Gembloux Agro-Bio Tech, University of Liege, Passage des Deportes-2, B-5030 Gembloux, Belgium (F. Francis).

E-mail addresses: Frederic.Francis@uliege.be (F. Francis), Virginie.Doyen@chu-brugmann.be (V. Doyen), France.Debaugnies@chu-brugmann.be (F. Debaugnies), gabriel.mazzucchelli@uliege.be (G. Mazzucchelli), r.caparros@uliege.be (R. Caparros), t.alabi@uliege.be (T. Alabi), christophe.blecker@uliege.be (C. Blecker), e.haubruge@uliege.be (E. Haubruge), francis.corazza@lhub.ulb.be (F. Corazza).
} 
were planned focusing on well known allergens already detected in several Arthropods. The development of a proteomic approach based on the extraction of particular group of allergens and on the use of reactive sera is one way to target active proteins. Then, particular immunoreactive candidates from one insect species can also be extracted from other taxa. The cross reactivity of individual human sera toward similar proteins from different edible entomological sources can be assessed. In this perspective, the aim of the present study was to investigate arginine kinase known as allergens mainly from shrimps and to investigate the cross-reactivity of these allergens taken from $A$. domesticus and T. molitor. For that purpose, proteomics was developed on whole edible insect samples but also on purified targeted arginine kinase fractions from these two edible hexapod species. Proteins were identified by Maldi-Tof after separation by gel electrophoresis coupled with mass spectrometry. Cross-reactivity of arginine kinase from different sources was discussed in relation to further use of edible insects in food in Western countries.

\section{Material and methods}

\subsection{Insect materials}

Insects were reared in containers maintained in a standardized room under a constant temperature $\left(25 \pm 1{ }^{\circ} \mathrm{C}\right)$ and relative humidity (65 $\pm 5 \%$ ). Tenebrio molitor was fed with a mixture of wheat flour, bran and yeast. After $24 \mathrm{~h}$ of starvation, insects were either used fresh for protein purification or frozen to kill them before cooking in an oven at $200{ }^{\circ} \mathrm{C}$ for $10 \mathrm{~min}$. This preparation was selected to correspond to one of the most frequent conditions these insects are consumed in Europe. Acheta domesticus were reared under similar feeding conditions but also providing water in appropriate aerated plastic cages $(60 \times 40 \times 40 \mathrm{~cm})$ where cardboards were set to increase surface/volume ratio.

After cooking, insect samples were ground to obtain a powder that was resuspended in $50 \mathrm{mM}$ Tris buffer at $\mathrm{pH} 7.0$ including a protease inhibitor cocktail (Promega) to extract the soluble proteins. A centrifugation was applied at $15,000 \mathrm{~g}, 15 \mathrm{~min}, 4^{\circ} \mathrm{C}$ and an aliquote of the supernatant was loaded on the SDS-PAGE.

\subsection{Arginine kinase partial purification}

Fresh insects (60 g of Tenebrio molitor or Acheta domesticus) were frozen and ground in liquid nitrogen. The obtained powders were suspended in $150 \mathrm{ml}$ of Tris $\mathrm{HCl} 50 \mathrm{mM}$; $0.2 \mathrm{mM}$ DTT; $1 \mathrm{mM}$ PMSF with a protease inhibitor cocktail (Promega). After overnight shaking at $4{ }^{\circ} \mathrm{C}$, the sample was centrifuged $20 \mathrm{~min}$ at $12,000 \mathrm{~g}$ and $4^{\circ} \mathrm{C}$. The supernatant was collected for further ammonium sulfate precipitation based on a three-step procedure $\left(30 \%, 70 \%\right.$ and $90 \%$ of $\left.\left(\mathrm{NH}_{4}\right)_{2} \mathrm{SO}_{4}\right)$. For each concentration, one hour shaking at $4{ }^{\circ} \mathrm{C}$ was followed by a $20 \mathrm{~min}$ centrifugation at $12,000 \mathrm{~g}$ and $4{ }^{\circ} \mathrm{C}$ to collect the protein pellet and add next amount of $\left(\mathrm{NH}_{4}\right)_{2} \mathrm{SO}_{4}$. Each pellet was suspended in Tris-HCl $20 \mathrm{mM} \mathrm{pH} 8.0$ buffer and dialyzed overnight in 50 times the volume. The sample was then filtered at $0.45 \mu \mathrm{m}$. A Q Sepharose FF ion exchange chromatography was used on an Akta system (GE) using the dialyzed $\left(\mathrm{NH}_{4}\right)_{2} \mathrm{SO}_{4} 30-70 \%$ fraction from the previous step. After washing and equilibration with Tris- $\mathrm{HCl} 20 \mathrm{mM}$ pH 8.0, $10 \mathrm{mM}$ 2-ME, $0.1 \mathrm{mM}$ EDTA buffer, protein sample was loaded and a continuous gradient from 0 to $1 \mathrm{M} \mathrm{NaCl}$ was applied at a $1 \mathrm{ml} / \mathrm{min}$ flow rate to collect $1 \mathrm{ml}$ fractions.

\subsection{Protein identification}

Excised spots from 1D gel bands were designated for the Proteineer dp automated Digester (Bruker, Bremen, Germany). Briefly, gel pieces were washed with three incubations in $100 \%$ of $50 \mathrm{mM}$ ammonium bicarbonate, followed by incubations in a 1:1 mixture of acetonitrile (ACN) and $50 \mathrm{mM}$ ammonium bicarbonate. Two additional washes were performed with $100 \%$ acetonitrile to dehydrate the gel. Gel pieces were first soaked in freshly activated trypsin (Porcine, Proteomics Grade, Roche) at $8{ }^{\circ} \mathrm{C}$ for $30 \mathrm{~min}$, and later subjected to protein trypsinization for $3 \mathrm{~h}$ at $30^{\circ} \mathrm{C}$. Peptide extractions were performed with $10 \mu \mathrm{L}$ of $1 \%$ formic acid (FA) for $30 \mathrm{~min}$ at $20^{\circ} \mathrm{C}$. Protein digests $(3 \mu \mathrm{L})$ were adsorbed for $3 \mathrm{~min}$ on pre-spotted Anchorchips (R) using the Proteineer dp automaton. Spots were washed on-target using $10 \mathrm{mM}$ ammonium dihydrogen phosphate in $0.1 \%$ trifluoroacetic acid (TFA) and desalted after washing with MilliQ water (Millipore). High throughput spectra were acquired using an Ultraflex II MALDI Mass Spectrometer (Bruker) in positive reflectron mode with close calibration enabled. Successful spectra were summed, treated in line with an automated SNAP algorithm using Flex Analysis 2.4 software (Bruker), and subsequently submitted in batch mode to the Biotools 3.0 software suite (Bruker) with an in-house hosted MASCOT search engine (www. MatrixScience.com). The public National Center for Biotechnology Information (NCBI) non-redundant database was used. A mass tolerance of $100 \mathrm{ppm}$ with close calibration and one missing cleavage site were allowed. Partial oxidation of methionine residues and complete carbamylation of cystein residues were considered. The probability score calculated by the software was used as a criterion for correct putative identification.

\subsection{Sera collection and preparation}

The sera used in this study were collected among the entomological laboratory staff at Gembloux Agro-Bio Tech - University of Liège according to volunteer willingness. Thirty-one people from 23 to 51-yearold contributed. All of them are continuously (several days per week) in direct contact with different kind of live insects according to their research topic and need to maintain insect rearing. They were subjected to a quantitative serum Immunoglobulin-E (IgE) level and skin test with edible insect allergens. Information on patients related to sera positive responses are presented in Table 1. The sera utilization authorization was approved by the Institutional Board at Brugman Hospital University Center in Brussels. Each individual serum was used individually for immunological tests.

\subsection{SDS-PAGE and immunoblotting}

For analytical SDS/PAGE, samples were diluted 1:2 (v:v) with a solubilizer (1\% SDS; $0.02 \%$ bromophenol; $1 \% \beta$-mercaptoethanol in Laemmli sample buffer) and boiled for $3 \mathrm{~min}$ before electrophoresis. Proteins and molecular weight standards were loaded in a stacking gel (3.5\% acrylamide, $0.5 \mathrm{M}$ Tris-HCl $\mathrm{pH}$ 6.8). Proteins were dissociated in a separation gel $(12.5 \%$ acrylamide; $0.01 \%$ SDS; $1.5 \mathrm{M}$ Tris- $\mathrm{HCl} \mathrm{pH}$ 8.8). Electrophoresis was carried out in Laemmli running buffer $(0.2 \mathrm{M}$ glycine; $0.1 \%$ SDS; $0.025 \mathrm{M}$ Tris, $\mathrm{pH} 8.3$ ), at $100 \mathrm{~V}$ and $50 \mathrm{~mA}$ for $2 \mathrm{~h}$ in a S-lab gel system (BioRad). The LMW-SDS Marker Kit (GE Healthcare)

Table 1

Information on entomological staff related to positive responses in immunoblots.

\begin{tabular}{llllllll}
\hline Ad N $^{\circ}$ & Tm N $^{\circ}$ & Sex & Age & Total IgE & Mite & Mealworm & Shrimp \\
\hline 8 & & M & 29 & 131.0 & $\mathbf{2 6 . 2 0}$ & $<0.01$ & 0.03 \\
9 & & F & 27 & 321.0 & $\mathbf{0 . 6 4}$ & $<0.01$ & $\mathbf{0 . 1 3}$ \\
10 & & F & 29 & 67.2 & $\mathbf{5 . 7 9}$ & $\mathbf{0 . 1 5}$ & $\mathbf{0 . 5 3}$ \\
11,12 & 15 & M & 24 & 56.6 & $\mathbf{0 . 1 1}$ & $<0.01$ & 0.04 \\
& 14 & M & 27 & 59.2 & $\mathbf{0 . 3 0}$ & 0.03 & 0.03 \\
& 16 & M & 25 & 176.0 & 0.08 & 0.00 & 0.05 \\
& 17 & M & 25 & 47.8 & $\mathbf{1 4 . 1 0}$ & 0.01 & 0.08 \\
& 18 & M & 29 & 97.5 & $\mathbf{5 . 8 0}$ & $\mathbf{5 . 6 0}$ & $\mathbf{3 . 5 0}$ \\
\hline
\end{tabular}

Ad $\mathrm{N}^{\circ}$ and $\mathrm{Tm} \mathrm{N}^{\circ}$ are related to Acheta domesticus columns and Tenebrio molitor columns on Fig. 2B and C respectively. Bold numbers are related to positive sensitivity to specific allergen (with quantification level threshold at $0.1 \mathrm{kU} / \mathrm{L}$ ). All people were sensitized but were not symptomatic for insect allergens. 
was used for molecular weight standards. The gels were directly stained with colloidal blue or used for further western blotting using a TransBlot $^{\circledR}$ Turbo $^{\mathrm{TM}}$ Transfer system (Biorad) for $10 \mathrm{~min}$ using nitrocellulose membrane.

After washing the membranes three times with distilled water, a $2 \mathrm{~h}$ blocking step at room temperature was applied under shaking with a PVP $2 \%$, Tween $0.1 \%$, TBS $(0.15 \mathrm{M} \mathrm{NaCl}, 0.05 \mathrm{M}$ Tris $\mathrm{HCl} \mathrm{pH} 7.6)$ solution. Then, overnight incubation at $4{ }^{\circ} \mathrm{C}$ was performed with primary antibody from individual patient (1/10 dilution rate). After 3 successive 10 min washing with TBS Tween buffer, the membrane was incubated with secondary antibody coupled with alcaline phosphatase (goat antihuman IgE HRP, KPL at 1/2000 dilution) for $5 \mathrm{~h}$ at room temperature. After a further 3 successive $10 \mathrm{~min}$ washing with TBS buffer, a 5 to 10 min revelation with NBT/BCIP solution (KPL) was performed.

\section{Results}

Beside protein extracted from whole insects, liquid chromatography technique was performed to separate targeted allergens from both selected insect species. The elution profiles of the arginine kinase from $A$. domesticus and T. molitor on ion exchange chromatography column are presented in Fig. 1. One main peak was observed first on both chromatographs even with a short delay for the mealworm sample. Fractions corresponding to these peaks of arginine kinase were collected

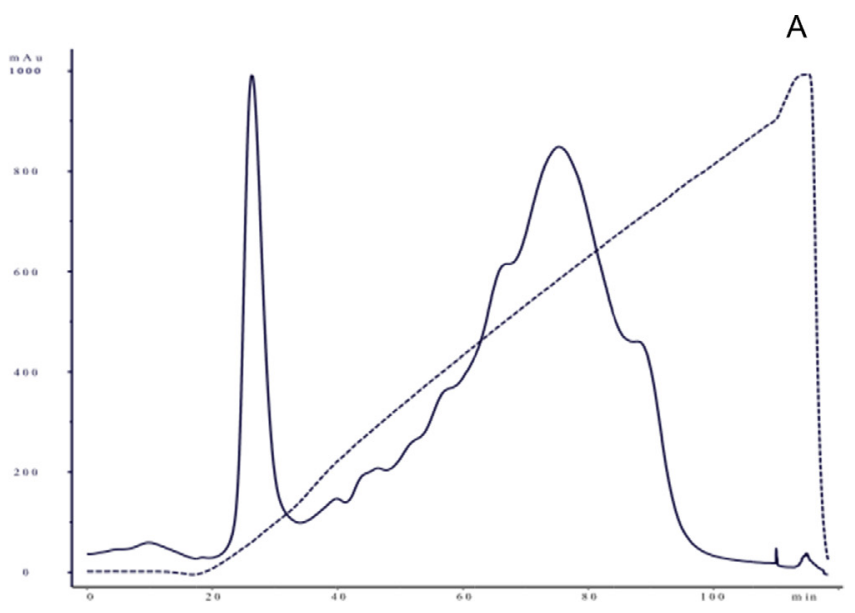

B

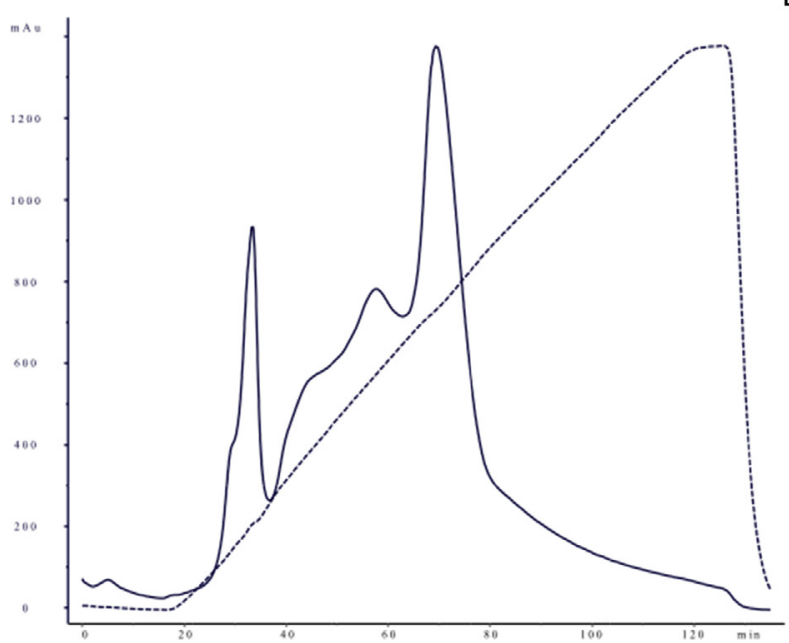

Fig. 1. Elution profiles of arginine kinase from Acheta domesticus (A) and Tenebrio molitor (B) on Q Sepharose ion exchange chromatography column Full lines are $\mathrm{UV}$ absorbance and dot lines $\mathrm{NaCl}$ gradient, first peak corresponded to argine kinase (fractions $22-32$ and $26-36$ for A and B respectively). and loaded on 1D SDS-PAGE gels. Different patterns were observed: two bands for A. domesticus at $40 \mathrm{kDa}$ (main one) and a minor at $14 \mathrm{kDa}$ (pool of fractions 22-32) and two bands of similar intensities at 17 and $25 \mathrm{kDa}$ for T. molitor (pool of fractions 26-36) (Fig. 2A). All these bands were cut from the electrophoresis gel to be analyzed in mass spectrometry. Three of them were identified as arginine kinase while one was related to a MAP kinase (Table 2).

The immunoblotting results revealed different responses according to the tested arginine kinase source samples (Fig. 2B and C). Systematic positive responses were obtained with individual sera against samples related to A. domesticus for both whole insect protein extracts and the purified arginine kinase (lines 8-12 from Fig. 2B). A diversity of bands was observed for samples related to $T$. molitor whole protein extract corresponding to non-specific responses (lines 14-17 from Fig. 2C). Nevertheless, light positive responses were obtained on bands at 25 and $17 \mathrm{kDa}$. Individual sera toward the partially purified arginine kinase mainly showed a $37 \mathrm{kDa}$ band and very light responses for $25 \mathrm{kDa}$. Different responses for arginine kinases were observed depending on the insect species source and individual selected serum.

\section{Discussion}

Most information on insect allergens are related to contact and injection exposures, such as for people in contact with cockroaches, mealworm rearing or exposed to venoms from bees and wasps (Barre et al., 2014). Few studies have focused on allergenicity related to edible insect ingestion. Nevertheless, some common allergenic molecules are cited in insects (Broekman et al., 2015; Burton \& Zaccone, 2007; Phillips \& Burkholder, 1995) and for a review on allergens in insects, a recent paper from Caparros Megido et al. (2015) could be recommended. As insects are closely related to crustacean and mites, panallergen molecules are generally incriminated when reactions and cross allergies occur (Barre et al., 2014; Broekman et al., 2017). Among potential targeted molecules, chitin, which constitutes up a large part of the arthropod exoskeleton, may have a role in the development of crossreactivity between mites, crustaceans and insects. Although the high prevalence of asthma among workers in contact with chitinous substances points towards an allergenic role of chitin, the experimental data are currently conflicting regarding the association between chitin and asthma (Brinchmann et al., 2011). Also, a recent study on allergic risks of ingesting edible insects has demonstrated a functional IgE crossreactivity of patients allergic to mites and crustaceans with T. molitor protein isolates. Main isolated proteins from T. molitor were a myosin heavy chain, alpha-amylase, tropomyosin and arginine kinase, many of them were known allergens from lobster, shrimp and mite (Verhoeckx et al., 2013).

Our study focused on arginine kinase from the two-main available edible insects species in Europe, T. molitor and A. domesticus. Arginine kinase is a phosphotransferase that plays a critical role in energy metabolism and the immune response (Yao, Ji, Kong, Wang, \& Xiang, 2009) and has been identified and characterized as an allergen in many species of shrimps such as the black tiger shrimp (Yu, Lin, Chiang, \& Chow, 2003), the pacific white shrimp (Garcia-Orozco, AispuroHernandez, Yepiz-Plascencia, Calderón-de-la- Barca, \& Sotelo-Mundo, 2007), the banana shrimp (Khanaruksombat, Srisomsap, Punyarit, \& Phiriyangkul, 2014) but also in silkworm larvae (Liu et al., 2009) and in some crickets (Yadzir et al., 2012). Also, the preparation of insect extracts influences the allergen solubility and further immunoblot differences in IgE binding between processed and unprocessed extracts. Thermal processing did not lower allergenicity but clearly changed solubility of mealworm allergens. Broekman et al. (2015) found different protein profiles after heat processing in all tested extracts. Protein bands less than $25 \mathrm{kDa}$ were more intense in all heat-processed samples compared to the raw extracts. In the latter, a protein band at $40 \mathrm{kDa}$ was identified as an arginine kinase. After processing, this band becomes more pronounced at a slightly higher molecular weight. A 


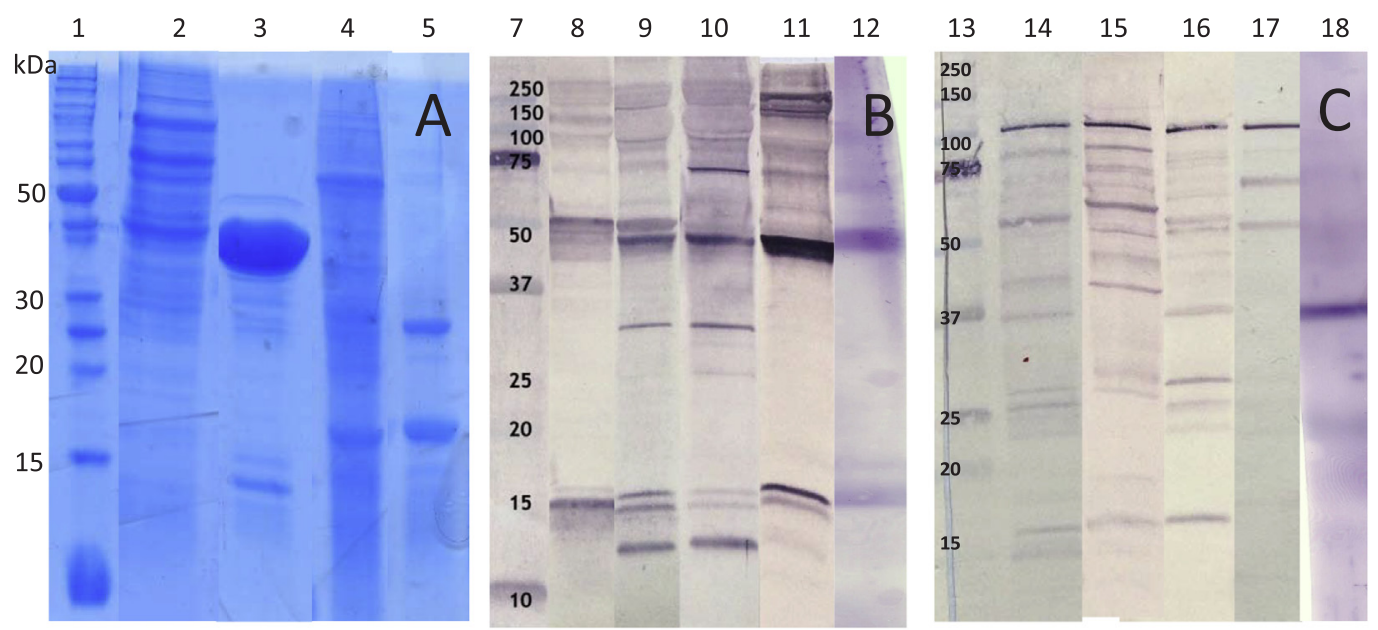

A: Molecular weight markers (1), Acheta domesticus total protein extract and purified arginine kinase (2-3), Tenebrio molitor total protein extract and purified arginine kinase (4-5) on SDS-PAGE gel.

B: Molecular weight markers (7), Acheta domesticus total protein extracts using 4 different patient sera (8-11) and purified arginine kinase (12) immunodetections on western blot membrane

C: Molecular weight markers (13), Tenebrio molitor total protein extract using 4 different patient sera (14-17) and purified arginine kinase (18) immunodetections on western blot membrane

Fig. 2. SDS-PAGE gel (A) and immunoblots (B-C) of whole edible insects (Acheta domesticus and Tenebrio molitor) whole protein extracts and purified arginine fractions from both species.

Table 2

Proteins identified from SDS-PAGE bands related to arginine kinase purification from Acheta domesticus and Tenebrio molitor.

\begin{tabular}{|c|c|c|c|c|c|c|c|c|}
\hline Picked bad sizes (kDA) & Protein identification & Organism & Accession number & Protein MW & PI value & Mowse score & MS coverage & Peptide numbers \\
\hline 40 & Arginine kinase & Acromyrmex echinatior & gi|332018357 & 40,032 & 5.9 & 110 & 42 & 21 \\
\hline 14 & Arginine kinase & Toxonotus cornutus & gi|228014792 & 18,838 & 8.2 & 50 & 23 & 4 \\
\hline \multicolumn{9}{|l|}{ Tenebrio molitor } \\
\hline 25 & Arginine kinase & Calosoma scrutator & gi|189064042 & 28,556 & 6.3 & 108 & 33 & 11 \\
\hline 17 & MAP kinase & Tribolium castaneum & gi|1004401433 & 11,138 & 9.7 & 56 & 45 & 5 \\
\hline
\end{tabular}

band of $27 \mathrm{kDa}$ was identified to be an arginine kinase from Xylosandrus crassiusculus (Broekman et al., 2015). In this study, similar changes in arginine kinase patterns were observed from 14 to $40 \mathrm{kDa}$ depending on the insect species but also on the applied processes either oven heated insect extracts or raw material after partial purification.

After a first investigation on people regularly in contact with insect rearing (the staff of an entomological university laboratory), different sensitivities were observed according to the target insect species but in each case, a cross reactivity with crustacean and mite was observed (Mairesse et al., 2014). In a recent study, Broekman et al. (2016) selected 60 patients with shrimp allergy that never consumed mealworm proteins. ImmunoCAP related to arginine kinase mealworm was positive in 10 patients and 4 mainly recognized proteins with a molecular weight of less than $25 \mathrm{kDa}$. Here, no cross reactivity was observed comparing A. domesticus and T. molitor samples when using sera from entomological laboratory staff. Although $21 \%$ of tested people reacted to $A$. domesticus arginine kinase, no specific reaction was found which similar proteins from $T$. molitor. This observation was surprising but confirmed that cross-reactivity is not systematic. When comparing arginine kinases from both studied insects, evidence of variation between these proteins was observed and the conservation/homology between them seems to be weak. Even if arginine kinases show high identities ( $70 \%$ on average) and homology (90\% on average) of sequences, different groups can be identified (Barre et al., 2014). These sequence homologies seem to be associated with a high structural homology, suggesting the possibility of cross-reactivity between these proteins. Although allergic cross-reaction was already determined between different insect species (silkworm and cockroaches; Liu et al., 2009) for arginine kinase, our results showed that differences could occurred at a specific level as no cross-reactivity was observed between arginine kinases from A. domesticus and T. molitor. Even if high sequence homology should be indicative for cross-reactivity, it must be confirmed by additional tests such as IgE binding immunoblots (Broekman et al., 2017).

With the development of entomophagy in Western countries in the next few years, the allergic risks of edible insects will increase due to direct higher exposure. Although most of potential exposed persons that will feed on insects have very little risk of an allergic reaction, particular attention will have to be paid for cross-reactivity.

People already displaying seafood and mites allergies could be more sensitive when eating insects. Even if our study did not show a crossreactivity between $T$. molitor and $A$. domesticus arginine kinases, other proteins (e.g. amylase or tropomyosine) could induced such reactions but also cross-reactivity between/with arginine kinases from other species could potentially occurred. Further studies are still needed on edible insect-related allergies in order to identify, characterize and manage all the potential allergens from different edible insect species to ensure that edible insect could become a safe source of human food. Changes in allergenic capacity of insect allergen according to insect cooking or cracking should also be part of further investigations. Finally, as long as edible insect allergies are still poorly understood, 
strong caution should be applied and the potential allergenic risk of edible insects must be clearly labeled on insect-based food products.

\section{References}

Barre, A., Caze-Subra, S., Gironde, C., Bienvenu, F., Bienvenu, J., \& Rougé, P. (2014). Entomophagie et risque allergique. Revue française d'allergologie, 54, 315-321.

Barre, A., Velazquez, E., Delplanque, A., Caze-Subra, S., Bienvenu, F., Bienvenu, J., ... Rougé, P. (2016). Cross-reacting allergens of edible insects. Revue Francaise d'Allergologie, 56, 522-532.

Belluco, S., Losasso, C., Maggioletti, M., Alonzi, C. C., Paoletti, M. G., \& Ricci, A. (2013). Edible insects in a food safety and nutritional perspective: A critical review. Comprehensive Reviews in Food Science and Food Safety, 12, 296-313.

Brinchmann, B. C., Bayat, M., Brøgger, T., Muttuvelu, D. V., Tjønneland, A., \& Sigsgaard, T. (2011). A possible role of chitin in the pathogenesis of asthma and allergy. Annals of Agricultural and Environmental Medicine, 18, 7-12.

Broekman, H., Knulst, A. C., de Jong, G., Gaspari, M., den Hartog Jager, C. F., Houben, G. F., \& Verhoeckx, K. C. M. (2017). Is mealworm or shrimp allergy indicative for food allergy to insects? Molecular Nutrition \& Food Research, 61(9), 1601061.

Broekman, H., Knulst, A., den Hartog Jager, S., Monteleone, F., Gaspari, M., de Jong, G., ... Verhoeckx, K. (2015). Effect of thermal processing on mealworm allergenicity. Molecular Nutrition \& Food Research, 59, 1855-1864.

Broekman, H., Verhoeckx, K. C., den Hartog Jager, C. F., Kruizinga, A. G., Pronk-Kleinjan, M., Remington, B. C., ... Knulst, A. C. (2016). Majority of shrimp-allergic patients are allergic to mealworm. The Journal of Allergy and Clinical Immunology, 137(4), 1261-1263.

Burton, O. T., \& Zaccone, P. (2007). The potential role of chitin in allergic reactions. Trends in Immunology, 28, 419-422.

Caparros Megido, R., Alabi, T., Larreché, S., Louxinger, A., Haubruge, E., \& Francis, F. (2015). Risques et valorisation des insectes dans l'alimentation humaine et animale. Annales de la Société entomologique de France.

Caparros, R. M., Sablon, L., Geuens, M., Brostaux, Y., Alabi, T., Blecker, C., ... Francis, F. (2013). Edible insects acceptance by Belgian consumers: Promising attitude for entomophagy development. Journal of Sensory Studies, 29(1), 14-20.

FAO (2009) Available from: < http://www.fao.org/filead min/templates/wsfs/docs/ expert_paper/How_to_Feed_the_World_in_2050.pdf/ > .

FASCS (2013) Available from: < http://www afsca.be/ denreesalimentaires/insectes/.

Gahukar, R. (2011). Entomophagy and human food security. International Journal of Tropical Insect Science. 31, 129-144.
Garcia-Orozco, K. D., Aispuro-Hernandez, E., Yepiz-Plascencia, G., Calderón-de-la- Barca, A. M., \& Sotelo-Mundo, R. R. (2007). Molecular characterization of arginine kinase, an allergen from the shrimp Litopenaeus vannamei. International Archives of Allergy and Immunology, 144, 23e28.

Khanaruksombat, S., Srisomsap, C., Chokchaichamnankit, D., Punyarit, P., Phiriyangkul, P. (2014) Identification of a novel allergen from muscle and various organs in banana shrimp (Fenneropenaeus merguiensis).

Liu, Z., Xia, L., Wu, Y., Xia, Q., Chen, J., \& Roux, K. H. (2009). Identification and characterization of an arginine kinase as a major allergen from silkworm (Bombyx mori) larvae. International Archives of Allergy and Immunology, 150, 8-14.

Looy, H., Dunkel, F. V., \& Wood, J. R. (2013). How then shall we eat? Insect-eating attitudes and sustainable foodways. Agriculture and Human Values, 31, 131-141.

Mairesse, M., Debaugnies, F., Doyen, V., Ledent, C., Michel, O., Corazza, F., \& Francis, F. (2014). Risque allergique des insectes en alimentation humaine. Revue Française d'Allergologie, 54(3), 288.

Phillips, J., \& Burkholder, W. (1995). Allergies related to food insect production and consumption. Food Insects Newsletters, 8(1-2), 4.

Ramos-Elorduy, J. (2009). Anthropo-entomophagy: Cultures, evolution and sustainability. Entomological Research, 39, 271-288.

Rumpold, B. A., \& Schlüter, O. K. (2013). Potential and challenges of insects as an innovative source for food and feed production. Innovative Food Science \& Emerging Technologies, 17, 1-11.

Sogari, G., Menozzi, D., \& Mora, C. (2017). Exploring young foodies' knowledge and attitude regarding entomophagy: A qualitative study in Italy. International Journal of Gastronomy and Food Science, 7, 16-19.

van Huis, A. (2003). Insects as food in Sub-Saharan Africa. Insect Science and Its Application, 23, 163-185.

Verhoeckx, K., van Broekhoven, S., Gaspari, M., de Hartog-Jager, S., de Jong, G., Wichers, H., ... Knulst, A. (2013). House dust mite (Derp 10) and crustacean allergic patients may be at risk when consuming food containing mealworms proteins. Clinical and Translational Allergy, 3, 48.

Yadzir, Z. H., Misnan, R., Abdullah, N., Bakhtiar, F., Arip, M., \& Murad, S. (2012). Identification of the major allergen of Macrobrachium rosenbergii. Asian Pacific Journal of Tropical Biomedicine, 2, 50-54.

Yao, C. L., Ji, P. F., Kong, P., Wang, Z. Y., \& Xiang, J. H. (2009). Arginine kinase from Litopenaeus vannamei: Cloning, expression and catalytic properties. Fish Shellfish Immunology, 26, 553e558.

Yu, C. J., Lin, Y. F., Chiang, B. L., \& Chow, L. P. (2003). Proteomics and immunological analysis of a novel shrimp allergen. Journal of Immunology, 170, 445-453. 